\title{
Relação entre internações, óbitos por doenças do aparelho circulatório e estrutura dos serviços
}

\section{Relationship between hospitalizations, deaths and service structure}

\author{
Cleuzieli Moraes dos Santos ${ }^{1,2}$ (D) Crhistinne Cavalheiro Maymone Gonçalves ${ }^{3,4}$ (D), \\ Daniel Henrique Tsuha ${ }^{5}$ (D) Albert Schiaveto de Souza ${ }^{6,7}$ (D), Ana Rita Barbieri ${ }^{8,9}$ (D) \\ 'Universidade Federal de Mato Grosso do Sul (UFMS) - Campo Grande (MS), Brasil. \\ ${ }^{2}$ Secretaria de Saúde de Mato Grosso do Sul - Campo Grande (MS), Brasil. \\ ${ }^{3}$ Faculdade de Saúde Pública, Universidade de São Paulo (USP) - São Paulo (SP), Brasil. \\ ${ }^{4}$ Faculdade de Medicina, Universidade Federal de Mato Grosso do Sul (UFMS) - Campo Grande (MS), Brasil. \\ ${ }^{5}$ Coordenadoria Estadual de Regulação, Secretaria de Saúde de Mato Grosso do Sul - Campo Grande (MS), Brasil. \\ ${ }^{6}$ University of Oxford - Oxford, Inglaterra. \\ 7 Instituto de Biociências, Universidade Federal de Mato Grosso do Sul (UFMS) - Campo Grande (MS), Brasil. \\ ${ }^{8}$ Fundação Oswaldo Cruz - Rio de Janeiro (RJ), Brasil. \\ 9Instituto Integrado de Saúde, Universidade Federal de Mato Grosso do Sul (UFMS) - Campo Grande (MS), Brasil.
}

Como citar: Santos CM, Gonçalves CCM, Tsuha DH, Souza AS, Barbieri AR. Relação entre internações, óbitos por doenças do aparelho circulatório e estrutura dos serviços. Cad Saúde Colet, 2020;28(2):211-222. https://doi.org/10.1590/1414$462 \times 202000020476$

\section{Resumo}

Introdução: A estruturação dos serviços de saúde é um elemento fundamental para dar respostas à carga de doenças crônicas. Objetivo: Analisar a relação entre internações e óbitos pelas doenças do aparelho circulatório e a estrutura nos serviços de saúde. Método: Estudo transversal desenvolvido em estado do Centro-Oeste brasileiro, região de saúde composta de 17 municípios e 1.112.792 habitantes. Foram analisadas as dimensões demográfica, epidemiológica e de estrutura de saúde pela estatística descritiva e correlação linear de Pearson. Resultados: A taxa da mortalidade por doenças do aparelho circulatório foi de $29,49 \%$, superior aos achados no país e no Centro-Oeste. Houve correlação significativa entre internação por doenças isquêmicas do coração e número de leitos e entre internação por doenças do aparelho circulatório e número de serviços especializados para cada 10 mil habitantes. Conclusão: As diferenças encontradas entre internações e a existência dos serviços especializados e leitos indicam que a população que reside em municípios com estrutura de serviços complexa possui mais acesso aos serviços e às internações. Os resultados demonstram que deve haver mais cuidado na conformação e oferta de serviços na Rede de Atenção à Saúde para as doenças do aparelho circulatório, visando assegurar equidade no acesso da população aos serviços pactuados.

Palavras-chave: política de saúde; serviços de saúde; regionalização; equidade no acesso; doenças cardiovasculares.

\footnotetext{
Abstract

Background: The structuring of health services is a fundamental element to respond to the burden of chronic diseases. Objective: To analyze the relationship between hospitalizations and deaths due to diseases of the circulatory system and the structure in health services. Method: A cross-sectional study developed in the state of the Brazilian central-west was carried out with a health region composed of 17 municipalities and 1,112,792 inhabitants. The demographic, epidemiological and health structure dimensions were analyzed using descriptive statistics and Pearson's linear correlation. Results: The mortality rate due to diseases of the circulatory system was $29.49 \%$, higher than the findings in the country

Trabalho faz parte da pesquisa intitulada Rede de Atenção à Saúde para a hipertensão arterial na região de saúde de Campo Grande, Mato Grosso do Sul, apresentada em dezembro de 2015, no Programa de Mestrado Profissional Saúde da Família da Universidade Federal do Mato Grosso do Sul. Compõe o tema em estudo por um grupo de pesquisa (CNPq) Campo Grande (MS), Brasil.

Correspondência: Cleuzieli Moraes dos Santos. E-mail: enf_cleuzieli@hotmail.com

Conflito de interesses: nada a declarar.

Recebido em: Dez. 28, 2017. Aceito em: Jun. 20, 2019
}

Este é um artigo publicado em acesso aberto (Open Access) sob a licença Creative Commons Attribution, que permite uso, distribuição e reprodução em qualquer meio, sem restrições desde que o trabalho original seja corretamente citado. 
and in the center-west. There was a significant correlation between hospitalization for ischemic heart diseases and number of beds, and between hospitalization for diseases of the circulatory system and number of specialized services for each 10,000 inhabitants. Conclusion: The differences found between hospitalizations and the existence of specialized services and beds indicate that the population that lives in municipalities with a complex service structure has more access to services and hospitalizations. The results demonstrate that there must be greater care in the conformation and offer of services in the Health Care Network for diseases of the circulatory system, aiming to ensure equity in the population's access to the agreed services.

Keywords: health policy; health services; regionalization; equity in access; cardiovascular diseases.

\section{INTRODUÇÃO}

A cobertura de saúde é uma dimensão a ser observada pelos formuladores de políticas. Estudos apontam que o agravamento das condições de saúde tem como uma das causas a insuficiente disponibilidade de recursos para atender a todos, o que fere o entendimento de que $o$ acesso aos serviços de saúde é um direito humano universal ${ }^{1-3}$. A equidade no acesso é caracterizada pelo tratamento diferente de acordo com as necessidades de cada indivíduo ou comunidade e, apesar da sua relevância, identificam-se escassos estudos que analisam o acesso da população aos serviços de saúde e a alocação dos recursos em associação com a situação de saúde. Tais relações são fundamentais para a avaliação de políticas e a organização da assistência em redes de atenção $0^{4,5}$.

A relação entre condições de saúde da população e o acesso aos serviços suscitou a necessidade de propor intervenções para a carga das doenças crônicas, por meio da proposta de modelos assistenciais que levem em conta a permanência do usuário no sistema em diferentes condições ${ }^{3}$. No Brasil, com o cenário muito semelhante ao de outros países, as doenças crônicas correspondem, em média, de $70 \%$ a $72 \%$ das causas de mortes, tendo a proporção por doenças do aparelho circulatório mais impacto ${ }^{6,7}$.

Modelos que fazem a integração de diversas estruturas de saúde têm sido objeto de políticas públicas em países como Canadá, Suécia, Holanda e Dinamarca ${ }^{8}$, todos empenhados em delinear políticas de rede de atenção com base em serviços existentes ou a criação de soluções para contemplar locais distantes ${ }^{9}$. Como resposta à superação da fragmentação do cuidado, no Brasil, em 2010, definiu-se que as redes de atenção à saúde (RASs) seriam incorporadas às políticas como o desenho para a organização dos serviços de saúde. Após tal delineamento, a Rede de Atenção à Saúde das Pessoas com Doenças Crônicas foi criada em $2013^{10}$.

Compreendida como arranjos organizativos de ações e serviços de saúde, a RAS prevê ações administrativas que visam integrar serviços com diferentes densidades tecnológicas, alocados em instituições diversas com relações horizontalizadas que possam dar sustentação às ações assistenciais ${ }^{2,10,11}$. Segundo essa concepção, também difundida pela Organização Mundial da Saúde (OMS), no Brasil, as RASs são constituídas por cinco componentes: atenção primária à saúde (APS), pontos de atenção especializada, sistemas de apoio, sistemas logísticos e sistema de governança2,10,11.

A estrutura dos serviços de saúde é um elemento básico para a constituição de redes assistenciais que precisam dar respostas à crescente carga de doenças crônicas ${ }^{8}$ e, diante da proposta de reorganização dos serviços em redes, o objetivo deste estudo é analisar a relação entre internações e óbitos pelas doenças do aparelho circulatório e estrutura existente nos serviços de saúde.

\section{MÉTODO}

O presente estudo transversal foi desenvolvido segundo os dados dos sistemas de informação do Sistema Único de Saúde (SUS), disponíveis em portais da internet, com informações demográficas, epidemiológicas e de estrutura de serviços, considerando a força de trabalho, na região de saúde de Campo Grande, capital do estado de Mato Grosso do Sul, composta de 17 municípios e 1.112 .792 habitantes $^{12}$. 
A escolha dessa região de saúde justifica-se por concentrar um terço da população do estado de Mato Grosso do Sul, Brasil, e entre eles situa a capital do estado, com serviços especializados ambulatoriais e hospitalares de alta densidade tecnológica.

As informações foram obtidas, respectivamente, dos portais do Departamento de Informática do SUS (Datasus), da Rede Interagencial de Informações para a Saúde (RIPSA), do Cadastro Nacional dos Estabelecimentos de Saúde (CNES) e do Departamento de Atenção Básica (DAB) (Quadro 1).

Para o estudo, coletaram-se informações de 2014, considerando as dimensões: 1) população total; 2) mortalidade e internações hospitalares por doenças do aparelho circulatório; 3 ) estrutura (leitos hospitalares, serviços especializados, força de trabalho no âmbito do SUS e cobertura de atenção primária por meio da estratégia de saúde da família [ESF]).

Os dados foram analisados por meio do programa estatístico SigmaPlot, versão 12.5, considerando um nível de significância de $5 \%$. Utilizou-se o teste de correlação de Pearson ${ }^{13}$ para análise da taxa de mortalidade de cada município e a taxa de internação, correspondente a três agrupamentos de agravos da Classificação Internacional de Doenças (CID-10) (I10-I15; I20-I25; I60-169), e entre a taxa de mortalidade ou a taxa de internação do município e as variáveis de número de profissionais, leitos, serviços especializados e cobertura de ESF.

Quadro 1. Fontes e características das informações utilizadas. Mato Grosso do Sul, 2014

\begin{tabular}{|c|c|c|c|c|}
\hline Dimensão & Variável & $\begin{array}{c}\text { Fonte } \\
\text { consultada }\end{array}$ & $\begin{array}{l}\text { Origem dos } \\
\text { dados }\end{array}$ & Cálculo da variável \\
\hline Demografia & $\begin{array}{l}\text { Estimativa de população } \\
\text { total residente. }\end{array}$ & Datasus & IBGE & Número absoluto. \\
\hline \multirow[b]{2}{*}{ Mortalidade } & $\begin{array}{l}\text { Taxa de mortalidade } \\
\text { específica por doenças } \\
\text { do aparelho circulatório } \\
\text { (Capítulo IX). }\end{array}$ & \multirow[b]{2}{*}{ Datasus } & \multirow[b]{2}{*}{$\begin{array}{l}\text { SIM/MS/ } \\
\text { SVS/CGIAE }\end{array}$} & $\begin{array}{l}\text { Número de óbitos } \\
\text { de residentes por } \\
\text { doenças do aparelho } \\
\text { circulatório sobre } \\
\text { o total de óbitos } \\
\text { multiplicado por } 100 .\end{array}$ \\
\hline & $\begin{array}{l}\text { Taxa de mortalidade } \\
\text { específica pelos } \\
\text { agrupamentos das } \\
\text { CID-10: I10 a I15: doenças } \\
\text { hipertensivas; I } 20 \text { a I25: } \\
\text { doenças isquêmicas do } \\
\text { coração; I } 60 \text { a I 69: doenças } \\
\text { cerebrovasculares. }\end{array}$ & & & $\begin{array}{l}\text { Número de óbitos } \\
\text { de residentes pelos } \\
\text { agrupamentos das } \\
\text { CID específicos sobre } \\
\text { o total de óbitos } \\
\text { por doenças do } \\
\text { aparelho circulatório } \\
\text { multiplicado por } 100 \text {. }\end{array}$ \\
\hline \multirow[b]{2}{*}{ Internação } & $\begin{array}{l}\text { Taxa de internação } \\
\text { (morbidade hospitalar) } \\
\text { específica por doenças } \\
\text { do aparelho circulatório } \\
\text { (Capítulo IX). }\end{array}$ & \multirow[b]{2}{*}{ Datasus } & \multirow[b]{2}{*}{$\mathrm{SIH} / \mathrm{SUS}$} & $\begin{array}{l}\text { Número de } \\
\text { internações de } \\
\text { residentes por } \\
\text { doenças do aparelho } \\
\text { circulatório sobre o } \\
\text { total de internações } \\
\text { multiplicado por } 100 .\end{array}$ \\
\hline & $\begin{array}{l}\text { Taxa de internação } \\
\text { (morbidade hospitalar) } \\
\text { específica pelos } \\
\text { agrupamentos das } \\
\text { CID-10: I10 a I15: doenças } \\
\text { hipertensivas; I } 20 \text { a I25: } \\
\text { doenças isquêmicas do } \\
\text { coração; I } 60 \text { a I 69: } \\
\text { doenças cerebrovasculares }\end{array}$ & & & $\begin{array}{l}\text { Número de } \\
\text { internações de } \\
\text { residentes pelos } \\
\text { agrupamentos das } \\
\text { CID específicos sobre } \\
\text { o total de internações } \\
\text { por doenças do } \\
\text { aparelho circulatório } \\
\text { multiplicado por } 100 .\end{array}$ \\
\hline
\end{tabular}

Fonte: Datasus; IBGE; SIM/MS/SVS/CGIAE; SIH/SUS; CNES/DATASUS/SAS/MS; DAB/SAS/MS. Nota: a seleção da estrutura dos serviços cadastrados no CNES foi realizada com base na VI Diretrizes para Hipertensão Arterial (Sociedade Brasileira de Cardiologia, 2010), que não estabelece a obrigatoriedade, mas descreve a forma de condução dos casos 
Quadro 1. Continuação...

\begin{tabular}{|c|c|c|c|c|}
\hline Dimensão & Variável & $\begin{array}{c}\text { Fonte } \\
\text { consultada }\end{array}$ & $\begin{array}{l}\text { Origem dos } \\
\text { dados }\end{array}$ & Cálculo da variável \\
\hline \multirow{6}{*}{ Estrutura } & $\begin{array}{l}\text { Leitos hospitalares do SUS } \\
\text { - leitos clínicos/cirúrgicos, } \\
\text { unidade de terapia intensiva } \\
\text { (UTI) adulto tipos II e III e } \\
\text { UTI coronariana - unidade } \\
\text { coronariana (UCO) tipo II. }\end{array}$ & \multirow{5}{*}{\multicolumn{2}{|c|}{$\begin{array}{l}\text { CNES/Datasus/ } \\
\text { SAS/MS }\end{array}$}} & \multirow{5}{*}{$\begin{array}{l}\text { Número absoluto } \\
\text { sobre a população } \\
\text { do município } \\
\text { de residência } \\
\text { multiplicado por } \\
10 \text { mil habitantes. }\end{array}$} \\
\hline & $\begin{array}{l}\text { Serviços especializados } \\
\text { - SUS: atenção } \\
\text { cardiovascular/cardiologia } \\
\text { - intervencionista: } \\
\text { hemodinâmica, cirurgia } \\
\text { cardiovascular adulto } \\
\text { e cardiologia clínica, } \\
\text { serviço por laboratório }\end{array}$ & & & \\
\hline & $\begin{array}{l}\text { clínico: exames } \\
\text { bioquímicos, uroanálise e } \\
\text { hematológicos, serviço de } \\
\text { diagnóstico por imagem: } \\
\text { radiologia, ultrassonografia } \\
\text { e tomografia e serviço de }\end{array}$ & & & \\
\hline & $\begin{array}{l}\text { diagnóstico por métodos } \\
\text { gráficos dinâmicos: exame } \\
\text { eletrocardiográfico, } \\
\text { teste de Holter, teste } \\
\text { ergométrico e exame } \\
\text { eletroencefalográfico. }\end{array}$ & & & \\
\hline & $\begin{array}{l}\text { Classificação Brasileira } \\
\text { de Ocupações (CBO) } \\
\text { - SUS: médico clínico, } \\
\text { cardiologista, cardiologista } \\
\text { intervencionista, intensivista, } \\
\text { cirurgião cardiovascular, } \\
\text { cirurgião torácico, enfermeiro } \\
\text { e enfermeiro da ESF }\end{array}$ & & & \\
\hline & $\begin{array}{l}\text { Cobertura de estratégia de } \\
\text { saúde da família }\end{array}$ & \multicolumn{2}{|c|}{ DAB/SAS/MS } & $\begin{array}{l}\text { Número de equipes } \\
\text { da ESF implantadas } \\
\text { multiplicado por } \\
3.000 \text {, multiplicado } \\
\text { por100 sobre a } \\
\text { população residente }\end{array}$ \\
\hline
\end{tabular}

Fonte: Datasus; IBGE; SIM/MS/SVS/CGIAE; SIH/SUS; CNES/DATASUS/SAS/MS; DAB/SAS/MS. Nota: a seleção da estrutura dos serviços cadastrados no CNES foi realizada com base na VI Diretrizes para Hipertensão Arterial (Sociedade Brasileira de Cardiologia, 2010), que não estabelece a obrigatoriedade, mas descreve a forma de condução dos casos

Esta pesquisa foi aprovada pelo Comitê de Ética da Universidade Federal de Mato Grosso do Sul (Conep 466/2012), sob o protocolo de número 773.454, e recebeu financiamento da Fundação de Apoio ao Desenvolvimento do Ensino, Ciência e Tecnologia do Estado do Mato Grosso do Sul (FUNDECT)/ Conselho Nacional de Desenvolvimento Científico e Tecnológico (CNPq) nº6/2011 - Programa de Apoio a Núcleos Emergentes (Pronem).

\section{RESULTADOS}

Nesta região de saúde, do total de óbitos, em 2014, 29,49\% foram decorrentes de doenças do aparelho circulatório. Conforme demonstrado na Figura 1, as menores taxas de mortalidade, $14,29 \%$, ocorreram em Jaraguari e Bandeirantes e a maior, em Corguinho (36,84\%). 


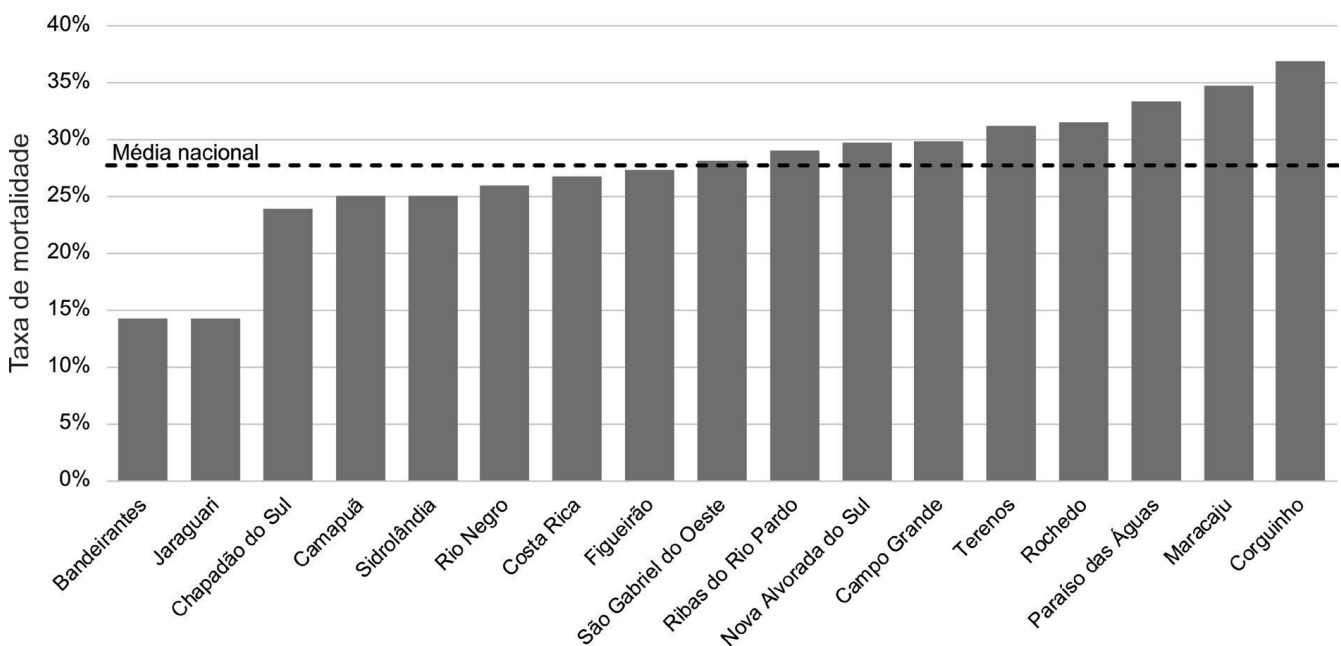

Figura 1. Taxa de mortalidade por doenças do aparelho circulatório (Capítulo IX) dos municípios da Região de Saúde de Campo Grande e a média nacional, 2014

As maiores taxas de internações hospitalares de todos os agravos das doenças do aparelho circulatório foram encontradas em Figueirão e em Rio Negro (14,30\% e 13,24\%, respectivamente), superiores às encontradas para essa região. Não houve registro dessas internações no município de Paraíso das Águas.

Os serviços da atenção primária alcançaram cobertura populacional superior a 75\% em 13 municípios e em três há diferenças na proporção de cobertura da população, havendo, na capital, menor proporção (38,55\%).

Os resultados de demografia, mortalidade e internação pelo agrupamento de agravos das doenças - capítulo das doenças do aparelho circulatório e de estrutura - estão apresentados na Tabela 1.

Não havia leitos em três municípios e nos demais se verificou uma distribuição que variava de 2,82 até 26,62 para cada 10 mil habitantes. Variações similares foram encontradas para serviços especializados, 1,61 a 19,97, e distribuição de profissionais, 7,38 a 25,45, para cada 10 mil habitantes, sem associação com o número de mortes ou internações por agrupamento de agravos das doenças do aparelho circulatório.

Observou-se correlação significativa entre internação por doenças isquêmicas do coração e número de leitos para cada 10 mil habitantes (Figura 2).

Também houve correlação entre internação por doenças do aparelho circulatório e número de serviços especializados para cada 10 mil habitantes (Figura 3).

\section{DISCUSSÃO}

A correlação encontrada entre a presença de leitos e as internações por doenças isquêmicas do coração, conforme demonstradas na Figura 2, e achados semelhantes na análise da relação entre serviços especializados e a taxa de internação na Figura 3 apontam uma desigualdade no acesso da população dos diferentes municípios aos serviços cardiovasculares especializados, evidenciando que os residentes nos municípios que possuem a estrutura especializada têm acesso diferenciado em relação aos demais.

Um dos objetivos do estabelecimento das RAS e, em particular da Rede de Atenção à Saúde das Pessoas com Doenças Crônicas, é organizar os serviços especializados sob a lógica da escala e, ao mesmo tempo, garantir o acesso de todas as pessoas a esses serviços. No entanto, os resultados apontaram diferenças no acesso aos serviços especializados e internações para os agrupamentos de doenças do aparelho circulatório analisados, sem diferenças estatísticas importantes para a oferta da assistência oferecida na atenção primária por meio da ESF.

Nas Américas, do total de mortes, $75 \%$ são decorrentes de doenças não transmissíveis, com a hipótese de aumento de 42,4\% até 2030, e, nos Estados Unidos, uma em cada cinco 


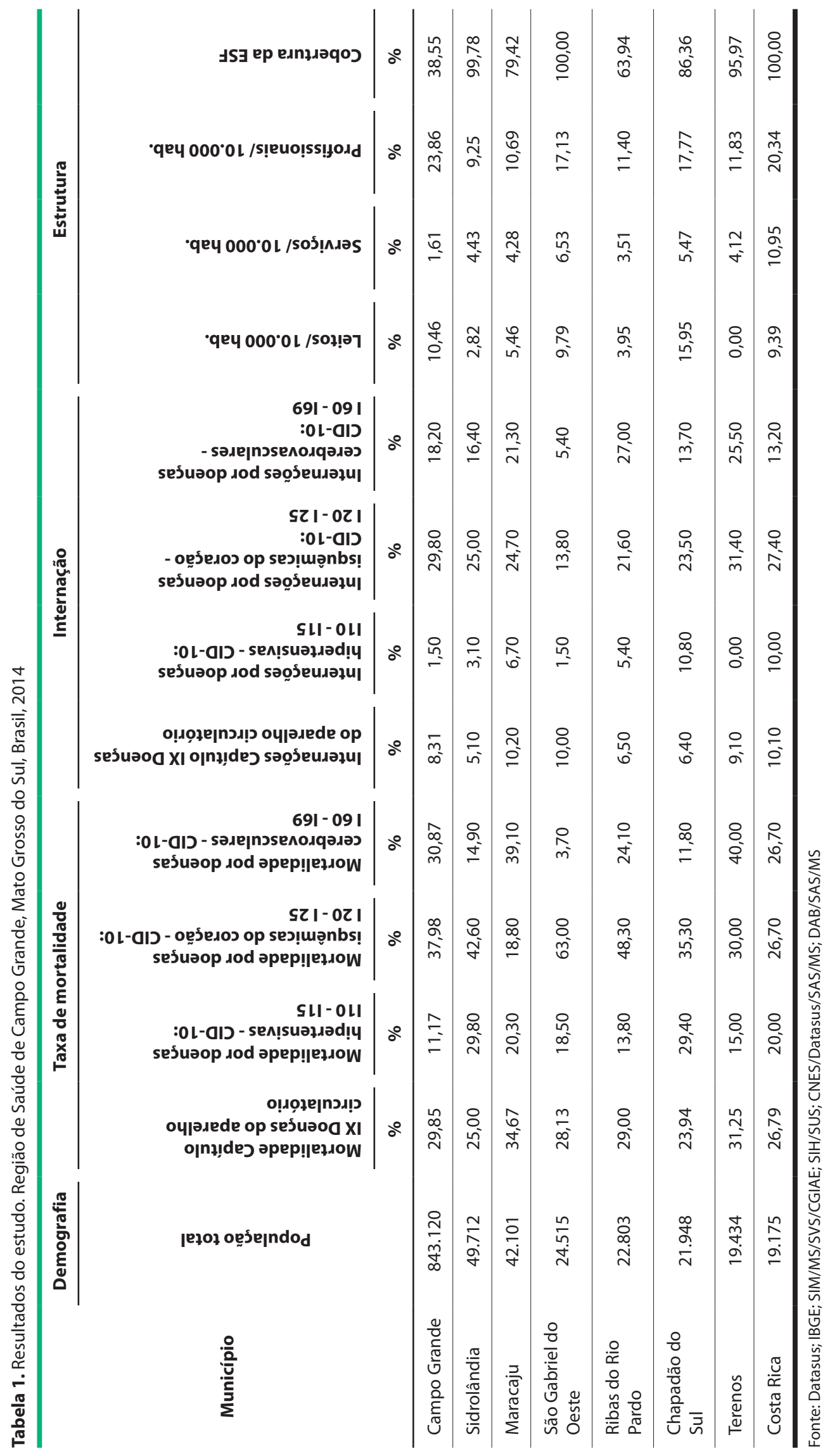



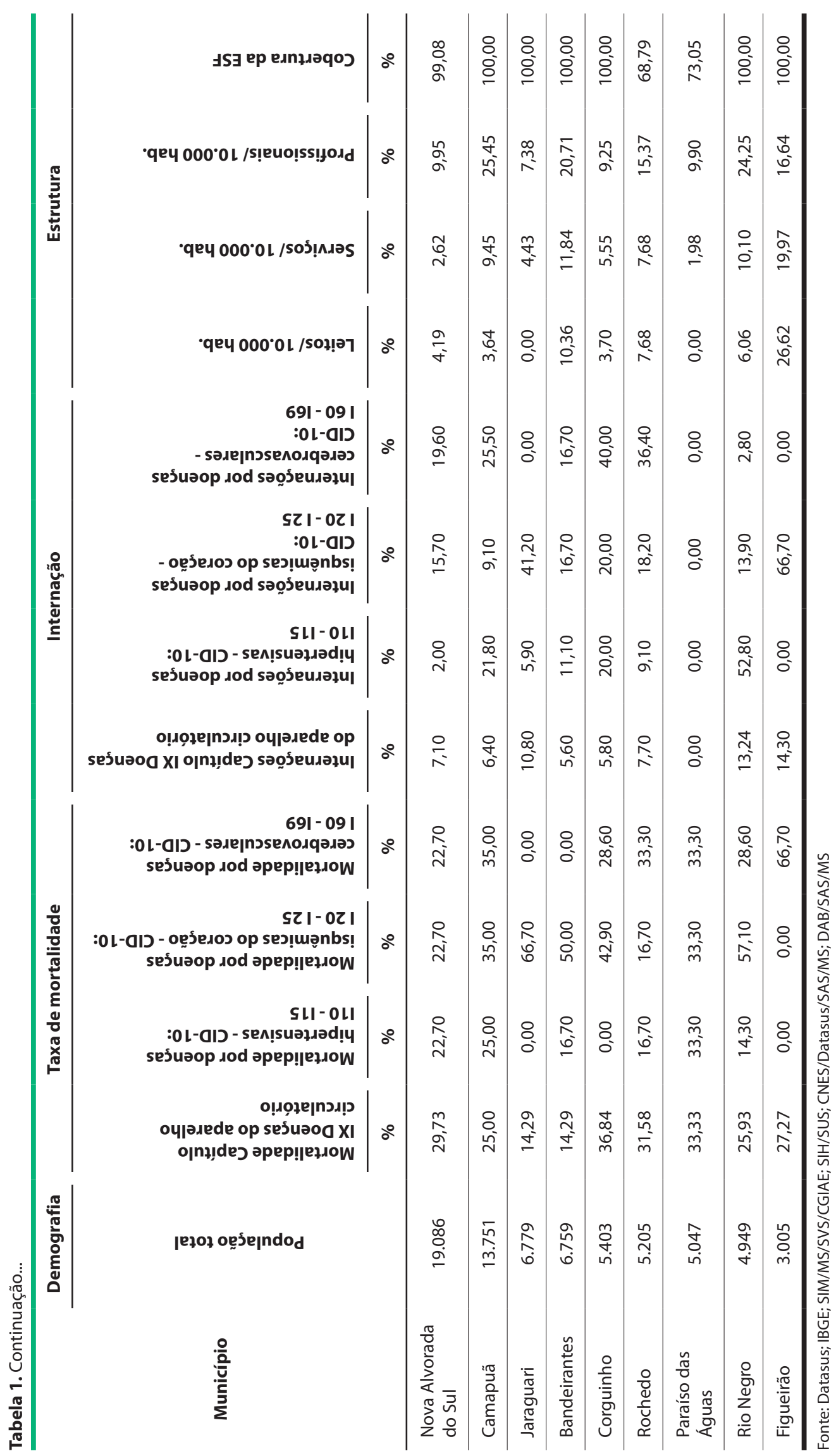


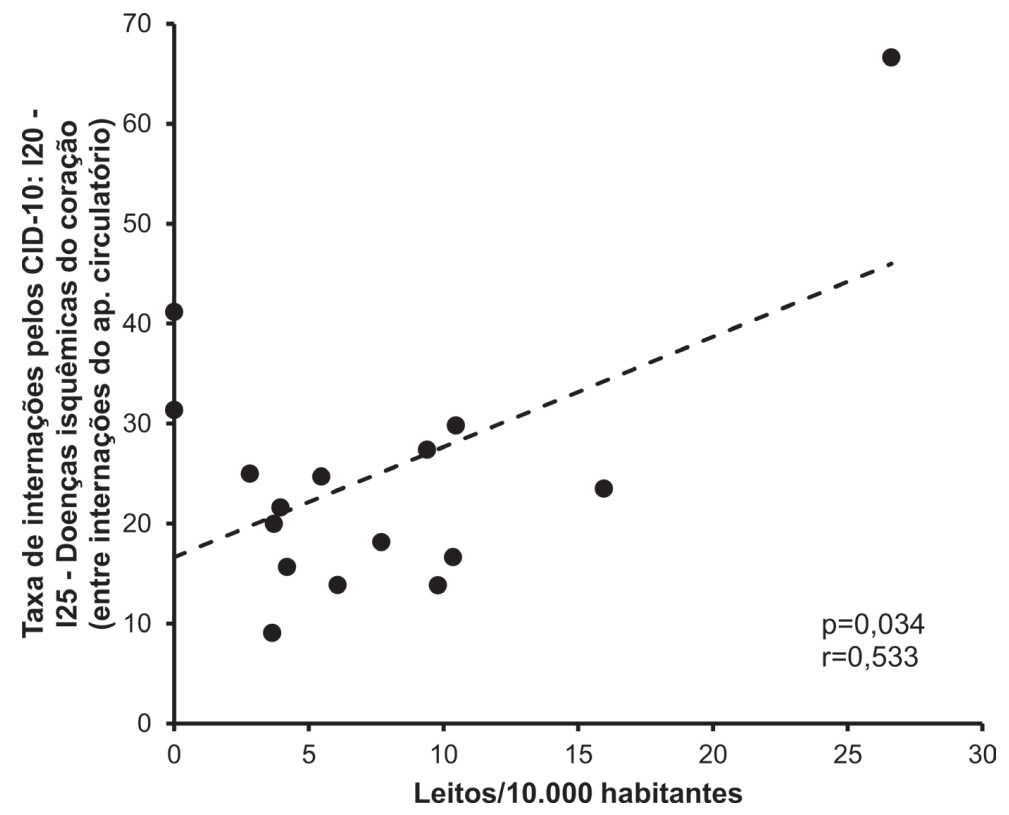

Figura 2. Correlação linear significativa positiva e moderada entre a taxa de internações por doenças isquêmicas do coração (entre as internações do aparelho circulatório) e o número de leitos para cada 10 mil habitantes. Região de Saúde de Campo Grande, Mato Grosso do Sul, Brasil, 2014. Nota: cada símbolo representa ambos os valores para um único município. A linha tracejada representa a linha de regressão linear. Valor de p no teste de correlação linear de Pearson. r: coeficiente de correlação linear

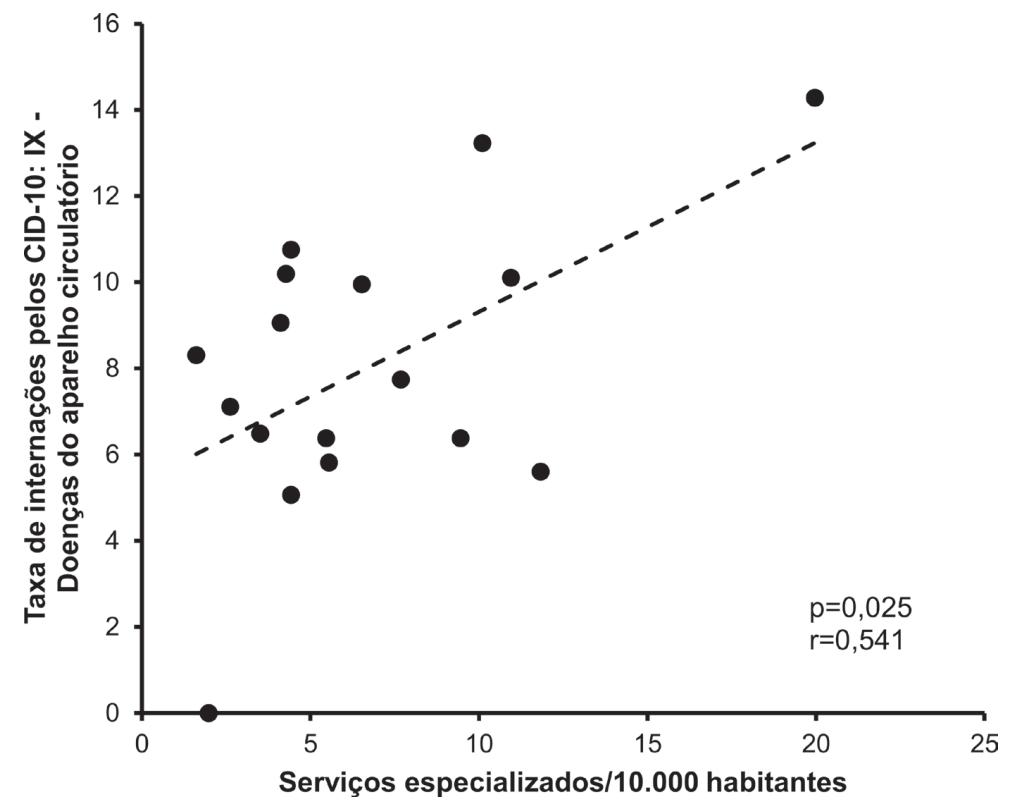

Figura 3. Correlação linear significativa positiva e moderada entre a taxa de internações por doenças do aparelho circulatório e a quantidade de serviços especializados para cada 10 mil habitantes. Região de Saúde de Campo Grande, Mato Grosso do Sul, Brasil, 2014. Nota: cada símbolo representa ambos os valores para um único município. A linha tracejada representa a linha de regressão linear. Valor de p no teste de correlação linear de Pearson. r: coeficiente de correlação linear

pessoas apresenta uma doença crônica, inclusive cardiovasculares ${ }^{14}$. Doenças do aparelho circulatório fazem parte do repertório de doenças consideradas sensíveis à atenção primária e constituem um marcador da qualidade da assistência, uma vez que a qualidade do cuidado pode evitar internações e óbitos precoces. 
Neste estudo, em 14 dos 17 municípios analisados, a cobertura da ESF é superior a 70\%, o que pode explicar a distribuição da mortalidade das doenças do aparelho circulatório sem diferenças estatísticas entre os municípios. Paradoxalmente, a atenção primária ao alcance da população em geral deveria ser uma estratégia relevante para ocorrer menos mortalidade por doenças cardiovasculares e tal resultado não foi observado. A presença de $100 \%$ de cobertura de ESF por si só não é suficiente para garantir a resolubilidade do cuidado no cenário brasileiro.

A partir de 2010, atendendo a recomendações de organismos internacionais, como a Organização Mundial da Saúde, e observando o desempenho dos sistemas de saúde de outros países, o Brasil dirigiu suas políticas públicas de saúde para a organização dos serviços em forma de redes de atenção como solução para a melhor distribuição dos serviços entre as populações. No entanto, estudos apontam importantes limitações no seu desenvolvimento, principalmente no que diz respeito à governança única, e nos sistemas integrados de informações que viabilizem o atendimento continuado dos pacientes ${ }^{15}$.

Nos municípios de Bandeirantes e Jaraguari, foi registrada uma taxa de mortalidade por doenças do aparelho circulatório em torno de 14,29 e nos demais ficou acima de 23,94. Para a região, a implantação de uma rede de atenção à saúde voltada às doenças cardiovasculares é imprescindível, dada a alta taxa de mortalidade por doenças do aparelho circulatório (29,49\% do total de óbitos), superior aos achados no Brasil, que registra $27,73 \%$, e na região Centro-Oeste, $26,53 \%$, respectivamente.

Muitos países, em particular os desenvolvidos, estruturam a rede de serviços de saúde sob o prisma da equidade, acessibilidade e sustentabilidade, permitindo a coordenação do cuidado segundo as necessidades de saúde da população ${ }^{16}$.

No que diz respeito à organização do sistema de saúde, na Espanha, o modelo em uso se aproxima muito da proposta brasileira de organizar os serviços em redes de atenção, com semelhança nos seus componentes que vão desde a atenção primária como porta de entrada até os níveis mais especializados. No entanto, lá se observa uma distribuição de recursos mais homogênea ${ }^{17}$.

No Brasil, o sistema de saúde não alcançou a equidade no acesso e essa desigualdade varia conforme o nível de atenção. Este estudo ratifica essa evidência. A desigualdade na utilização de serviços especializados ambulatoriais é mais evidente do que nos outros níveis de atenção, com forte influência da renda e da capacidade de compra de serviços privados de saúde ${ }^{4,18}$. Para que todos se beneficiem dos serviços especializados, recursos de apoio, como sistemas informatizados, transporte sanitário e financiamento, devem ser assegurados no momento do planejamento e de tomada de decisão.

Neste estudo, verificou-se a associação entre número de leitos e internações hospitalares para doenças isquêmicas e entre doenças do aparelho circulatório e serviços especializados. Nos países desenvolvidos, observa-se que há uma relação, em média, de três a seis leitos por mil habitantes, enquanto os achados na pesquisa apontam para menos de um leito por mil habitantes, fato que pode estar contribuindo para a alta mortalidade por doenças cardiovasculares em geral na região, apesar da presença da atenção primária no território como um todo.

Outro aspecto que chama a atenção é a distribuição desigual de leitos entre os municípios, inclusive com hospitais de pequeno porte e baixa complexidade para uma população que não alcança dez mil habitantes, como em Bandeirantes, Figueirão e Rio Negro. Neste aspecto, observa-se que países desenvolvidos que organizam seus sistemas de saúde baseados em eficiência têm reduzido a quantidade de hospitais de pequeno porte para investir em hospitais com maior número de leitos, o que otimiza os serviços e reduz os custos ${ }^{19}$.

Nesse sentido, a organização dos leitos hospitalares em maiores hospitais favorece a governança da rede de atenção, variável pouco desenvolvida na região, para a garantia do adequado acesso aos leitos por toda a população que deles necessita ${ }^{15}$.

Há evidências que apontam a necessidade de organizar os serviços de uma forma integrada em todos os níveis, segundo as peculiaridades da região. A integração dos serviços garante a prestação de um cuidado mais completo, que vai desde a promoção e prevenção ao tratamento e reabilitação dos casos demandados ${ }^{20}$. Em particular, o cuidado às pessoas 
com doenças crônicas é favorecido com a implantação de redes integradas, ou seja, redes de atenção com oferta de trabalho multiprofissional ${ }^{10}$.

Constata-se a concentração de recursos especializados de maior densidade tecnológica e força de trabalho em um município, a capital do estado (Tabela 1). O porte populacional do município, as realidades territoriais e as necessidades de saúde demandam a organização da rede de atenção com abrangência regional, sob a lógica da economia de escala, o que justifica sua concentração em apenas um município. Contudo, disso decorre a necessidade de articulação e pactuação locorregional, com mecanismos regulatórios definidos, para condução oportuna, integral, eficaz, eficiente e equânime dos $\operatorname{casos}^{21}$.

Há tendência de os profissionais de saúde trabalharem em municípios economicamente desenvolvidos. No entanto, a relação entre o número de profissionais e o acesso aos serviços não garante equidade no acesso ${ }^{1}$. A disponibilidade e a gestão da força de trabalho em saúde se constituem fatores decisivos e críticos para efetivar o sistema. No Brasil, a distribuição desigual de recursos profissionais, o deficiente contingente de médicos e outros profissionais, a oferta de especialidades não condizente com as necessidades, entre outros, merecem atenção e as políticas públicas nesse campo ainda estão incipientes. Reconhecer e planejar a força de trabalho consoante às necessidades de saúde deve compor a pauta para a organização das redes $^{22}$.

O maior desafio dos municípios para que possam se organizar em redes assistenciais eficientes e racionais é a busca de mecanismos que assegurem acesso aos serviços especializados. A regionalização é uma proposta que se efetiva com base em mecanismos regulatórios mais organizados e acessíveis ${ }^{23}$.

A regionalização, quando operacionalizada, possibilita uma distribuição mais equânime dos serviços e ações de saúde e potencializa o desenvolvimento do espaço locorregional ao reconhecer as dinâmicas do território regional que se relacionam com o processo saúde-doença ${ }^{24,25}$. O processo de regionalização, por meio da estruturação de regiões de saúde, entre outros aspectos, favorece e qualifica a gestão compartilhada, a integração da rede de serviços e a resolubilidade das necessidades de saúde ${ }^{23}$.

Pesquisadores apontam que a organização dos modelos de atenção à saúde baseada em RAS favorece, entre outras questões, a definição das atribuições de cada componente, como rede hospitalar, cuidados intensivos, implantação dos serviços extra-hospitalares, coordenação da atenção primária e conformação da rede de urgência e emergência, e facilita a avaliação ${ }^{25}$.

Em um documento publicado em 2014, a OMS apontou evidências de padrões diferentes de cobertura de serviços de saúde, de acordo com a renda, e sugere que as ações sejam universais e dedicadas à população em geral, sem restrições ${ }^{26}$. No Brasil, o sistema de saúde não alcançou a equidade no acesso e essa desigualdade varia conforme o nível de atenção. A desigualdade na utilização de serviços especializados ambulatoriais é mais evidente do que em outros níveis de atenção. $O$ atendimento de emergência é utilizado com mais frequência pela população de baixa renda, podendo ser consequência de barreiras de acesso à atenção primária, como a falta de médicos ou baixos níveis de resolubilidade ${ }^{4,18}$.

Este estudo apresenta algumas limitações. A primeira é o uso de dados secundários, que, mesmo utilizados para estudos transversais, podem apresentar falhas, sendo uma delas o registro incorreto da informação. A segunda é que sistemas de informações podem ter possíveis falhas nos cadastros e anotações de atendimento e condução dos casos. A taxa de mortalidade por doenças do aparelho circulatório suscita mais estudos na região e este estudo, em particular, pode contribuir para elucidar as barreiras de acesso, evidenciadas com os resultados apresentados. No entanto, apesar das limitações, informações produzidas por bancos de dados podem ser utilizadas porque são confiáveis desde que observados seus limites $^{27}$. Além disso, no processo de planejamento e de tomada de decisão, sistemas de informação em saúde devem ser utilizados ${ }^{28}$.

Os achados desta pesquisa apontam para a necessidade de relacionar a dimensão epidemiológica e de estrutura de serviços para a discussão da equidade do acesso das pessoas aos serviços de saúde. A ampliação da cobertura da ESF não é suficiente para reduzir óbitos e internações provocados pelas condições crônicas e, em particular, para os agrupamentos selecionados para este estudo. 
As associações encontradas entre internações, oferta de serviços especializados e populações residentes em diferentes municípios demonstram diferenças no acesso, privilegiando a população que reside nos municípios com mais oferta de assistência especializada.

Os gestores públicos de saúde no processo de tomada de decisão necessitam considerar os aspectos da estrutura existente dos serviços, sob a lógica da suficiência e distribuição, não unicamente de acordo com a oferta instalada, e sim das necessidades da população que podem ser indiretamente consideradas pelos indicadores de morbimortalidade.

Descrever a estrutura e oferta dos serviços segundo uma condição crônica de grande magnitude, como doenças do aparelho circulatório, em uma região, também favorece a reprodução dessa metodologia para outras regiões de saúde e agravos, otimizando, assim, a análise dos cenários que precedem a tomada de decisão para a implantação de outras redes de atenção à saúde.

\section{REFERÊNCIAS}

1. Chen R, Zhao Y, Du J, Wu T, Huang Y, Guo A. Health worforce equity in urban community health service of China. PLoS One. 2014;9(12):e115988. http://dx.doi.org/10.1371/journal.pone.0115988. PMid:25551449.

2. Castillo CHM, Garrafa V, Cunha T, Hellmann F. Access to health care as a human right in international policy: critical reflections and contemporary challenges. Cien Saude Colet. 2017;22(7):2151-60. http:// dx.doi.org/10.1590/1413-81232017227.04472017. PMid:28723997.

3. White F. Primary health care and public health: Foundations of Universal Health Systems. Med Princ Pract. 2015;24(2):103-16. http://dx.doi.org/10.1159/000370197. PMid:25591411.

4. Garcia-Subirats I, Lorenzo IV, Mogollón-Pérez AS, Paepe P, Silva MRF, Unger JP, et al. Determinantes del uso de distintos niveles asistenciales en el Sistema General de Seguridad Social en Salud y Sistema Único de Salud en Colômbia y Brasil. Gac Sanit. 2014;28(6):480-8. http://dx.doi.org/10.1016/j.gaceta.2014.05.010. PMid:25048392.

5. Santana P, Costa C, Marí-Dell'Olmo M, Gotsens M, Borrell C. Mortality, material deprivation and urbanization: exploring the social patterns of a metropolitan area. Int J Equity Health. 2015;14(1):55. http://dx.doi. org/10.1186/s12939-015-0182-y. PMid:26051558.

6. Brasil. Ministério da Saúde. Plano de ações estratégicas para o enfrentamento das doenças crônicas não transmissíveis (DCNT) no Brasil 2011-2022. Brasília, DF: Ministério da Saúde; 2011.

7. Malta DC, Moura L, Prado RR, Escalante JC, Schmidt MI, Duncan BB. Mortalidade por doenças crônicas não transmissíveis no Brasil e suas regiões, 2000 a 2011. Epidemiol Serv Saude. 2014;23(4):599-608. http:// dx.doi.org/10.5123/S1679-49742014000400002.

8. Contel JC, Muntané B, Camp L. La atención al paciente crónico en situación de complejidad: el reto de construir un escenario de atención integrada. Aten Primaria. 2012;44(2):107-13. http://dx.doi.org/10.1016/j. aprim.2011.01.013. PMid:21636176.

9. World Health Organization. Health 2020 A European policy framework and strategy for the 21 st century. Geneva:WHO; 2013.

10. Chueiri PS, Harzheim E, Gauche H, Vasconcelos LLC. Pessoas com doenças crônicas, as redes de atenção e a Atenção Primária à Saúde. Divulgação em Saúde para Debate. 2014;52:114-24.

11. Magalhães Júnior HM. Redes de Atenção à Saúde: rumo à integralidade. Divulgação em Saúde para Debate. 2014;52:15-37.

12. Datasus. Departamento de Informática do SUS. Informações de Saúde - TABNET [Internet]. 2014 [citado em 2015 jun 12]. Disponível em: http://www2.datasus.gov.br/DATASUS/index.php?area=02.

13. Espírito-Santo H, Daniel F. Calcular e apresentar tamanhos do efeito em trabalhos científicos (1): As limitações do $p<0,05$ na análise de diferenças de médias de dois grupos. Rev Portuguesa de Investigação Comportamental e Social. 2015;1(1):3-16. http://dx.doi.org/10.7342/ismt.rpics.2015.1.1.14.

14. Organización Panamericana de la Salud. Enfermedades no transmisibles en las Américas: construyamos un futuro más saludable. Washington, DC: OPAS; 2011.

15. Santos CM, Barbieri AR, Gonçalves CAM, Tsuha DH. Avaliação da rede de atenção ao portador de hipertensão arterial: estudo de uma região de saúde. Cad Saude Publica. 2017;33(5):e00052816. http:// dx.doi.org/10.1590/0102-311x00052816. PMid:28614449. 
16. World Health Organization. WHO global strategy on people-centred and integrated health services. World Health Organization: Geneva, Switzerland; 2015.

17. Lamata Cotanda F. Atención sanitária y rede de servicios. Madrid: Escuela Nacional de Sanidad; 2011 (Tema 2.9).

18. Teles AS, Coelho TCB, Ferreira MPS, Scatena JHG. Serviço de Atendimento Móvel de Urgência (SAMU) do Estado da Bahia: subfinanciamento e desigualdade regional. Cad Saude Colet. 2017;25(1):51-7. http:// dx.doi.org/10.1590/1414-462×201700010188.

19. Morikawa M. Economies of Scale and Hospital Productivity: An Empirical Analysis of Medical Area. Tokyo: RIETI. 2010. (Discussion Paper Series 10-E-050).

20. Veras RP, Caldas CP, Motta LB, Lima KC, Siqueira RC, Rodrigues RTSV, et al. Integration and continuity of Care in health care network models for frail older adults. Rev Saude Publica. 2014;48(2):1-8. http://dx.doi. org/10.1590/S0034-8910.2014048004941. PMid:24897058.

21. Chaves LDP, Jesus BJ, Ferreira JBB, Balderrama P, Tanaka OY. Avaliação de resultados da atenção aos agravos cardiovasculares como traçador do princípio de integralidade. Saude Soc. 2015;24(2):568-77. http://dx.doi.org/10.1590/S0104-12902015000200014.

22. Rizzotto MLF, Gil CRR, Carvalho M, Fonseca ALN, Santos MF. Força de trabalho e gestão do trabalho em saúde: revelações da Avaliação Externa do Programa Nacional de Melhoria do Acesso e da Qualidade da Atenção Básica no Paraná. Saúde Debate. 2014;38(N. esp.):237-51.

23. Santos L, Campos GWS. SUS Brasil: a região de saúde como caminho. Saude Soc. 2015;24(2):438-46. http:// dx.doi.org/10.1590/S0104-12902015000200004.

24. Contel FB. Os conceitos de região e regionalização: aspectos de sua evolução e possíveis usos para a regionalização da saúde. Saude Soc. 2015;24(2):447-60. http://dx.doi.org/10.1590/S010412902015000200005.

25. Costa LS, Gadelha CAG, Borges TR, Burd P, Maldonado J, Vargas M. A dinâmica inovativa para a reestruturação dos serviços de saúde. Rev Saude Publica. 2012;46(Suppl.):76-82. http://dx.doi.org/10.1590/ S0034-89102012000700011. PMid:23532313.

26. World Health Organization. Monitoring health inequality: An essential step for achieving health equity. Geneva:WHO; 2014.

27. Coeli CM, Pinheiro RS, Carvalho MS. Nem melhor, nem pior, apenas diferente. Cad Saude Publica. 2014;30(7):1363-5. http://dx.doi.org/10.1590/0102-311XPE010714. PMid:25166933.

28. Pinheiro ALS, Andrade KTS, Silva DO, Zacharias FCM, Gomide MFS, Pinto IC. Gestão da saúde: o uso dos sistemas de informação e o compartilhamento de conhecimento para a tomada de decisão. Texto Contexto Enferm. 2016;25(3):1-9. http://dx.doi.org/10.1590/0104-07072016003440015. 